\title{
On quasi-conformally flat and quasi-conformally semisymmetric generalized Sasakian-space-forms
}

\author{
D.G. PRAKASHA \\ Department of Mathematics, \\ Karnatak University, \\ Dharwad-580 003 Karnataka State, India. \\ prakashadg@gmail.com
}

\author{
H.G. NAGARAJA \\ Department of Mathematics \\ Central College Campus, \\ Bangalore University, Bangalore-560 001, \\ India. \\ hgnraj@yahoo.com
}

\begin{abstract}
The object of the present paper is to study quasi-conformally flat and quasi-conformally semisymmetric generalized Sasakian-space-forms.

\section{RESUMEN}

El objeto del artículo actual es estudiar formas de espacio Sasakian cuasi-conformacionales planas y cuasi-conformacionales generalizadas semisimétricas.
\end{abstract}

Keywords and Phrases: Generalized Sasakian-space-forms, quasi-conformally flat, quasi-conformally semisymmetric, Einstein manifold, scalar curvature.

2010 AMS Mathematics Subject Classification: 53C25, 53D15. 


\section{Introduction}

The notion of generalized Sasakian-space-forms was introduced and studied by Alegre et al [1] with several examples. A generalized Sasakian-space-form is an almost contact metric manifold $(M, \phi, \xi, \eta, g)$ whose curvature tensor is given by

$$
\begin{aligned}
R(X, Y) Z & =f_{1}\{g(Y, Z) X-g(X, Z) Y\} \\
& +f_{2}\{g(X, \phi Z) \phi Y-g(Y, \phi Z) \phi X+2 g(X, \phi Y) \phi Z\} \\
& +f_{3}\{\eta(X) \eta(Z) Y-\eta(Y) \eta(Z) X \\
& +g(X, Z) \eta(Y) \xi-g(Y, Z) \eta(X) \xi\}
\end{aligned}
$$

where $f_{1}, f_{2}, f_{3}$ are differentiable functions on $M$ and $X, Y, Z$ are vector fields on $M$. In such case we will write the manifold as $M\left(f_{1}, f_{2}, f_{3}\right)$. This kind of manifolds appears as natural generalization of the Sasakian-space-forms by taking:

$$
f_{1}=\frac{c+3}{4} \text { and } f_{2}=f_{3}=\frac{c-1}{4},
$$

where $\mathrm{c}$ denotes constant $\phi$-sectional curvature. The $\phi$-sectional curvature of generalized Sasakianspace-forms $M\left(f_{1}, f_{2}, f_{3}\right)$ is $f_{1}+3 f_{2}$. Moreover, cosymplectic space-forms and Kenmotsu spaceforms are also particular case of generalized Sasakian-space-forms. In the recent paper P. Alegre and A. Carriazo [2] studied contact metric and trans-Sasakian generalized Sasakian-space-forms. Generalized Sasakian-space-forms have been studied by several authors, viz., [5, 6, 10, 12,

In Riemannian geometry, many authors have studied curvature properties and to what extent they determined the manifold itself. Two important curvature properties are flatness and symmetry. As a generalization of local symmetric space, the notion of semisymmetric space [13] is defined as $R(X, Y) \cdot R=0$, where $R(X, Y)$ acts on $R$ as a derivation. In this connection, the conformal flatness and local symmetry of generalized Sasakian-space-forms was studied in [10]. Also in [6], generalized Sasakian-space-forms with vanishing projective curvature tensor and some symmetry properties have been considered. Motivated by these facts, in this paper we study the flatness and semisymmetry property of generalized Sasakian-space-form regarding the quasi-conformal curvature tensor.

The notion of the quasi-conformal curvature tensor was given by Yano and Sawaki [14]. According to them in a $(2 n+1)$-dimensional $(n>1)$ almost contact metric manifold the quasiconformal curvature tensor $\widetilde{C}$ is defined by

$$
\begin{aligned}
\widetilde{C}(X, Y) Z= & a R(X, Y) Z+b[S(Y, Z) X-S(X, Z) Y+g(Y, Z) Q X-g(X, Z) Q Y] \\
& -\frac{r}{2 n+1}\left(\frac{a}{2 n}+2 b\right)[g(Y, Z) X-g(X, Z) Y]
\end{aligned}
$$

where $a$ and $b$ are constants and $R, S, Q$ and $r$ are the Riemannian curvature tensor of type $(1,3)$, the Ricci tensor of type $(0,2)$, the Ricci operator defined by $g(Q X, Y)=S(X, Y)$ and the scalar 
curvature of the manifold respectively. If $a=1$ and $b=-\frac{1}{2 n-1}$, then (1) takes the form

$$
\begin{aligned}
C(X, Y) Z= & R(X, Y) Z-\frac{1}{2 n-1}[S(Y, Z) X-S(X, Z) Y+g(Y, Z) Q X-g(X, Z) Q Y] \\
& +\frac{r}{(2 n)(2 n-1)}[g(Y, Z) X-g(X, Z) Y]=C(X, Y) Z
\end{aligned}
$$

where $\mathrm{C}$ is the conformal curvature tensor [8]. Thus conformal curvature tensor is a particular case of quasi-conformal curvature tensor.

A manifold ( $M, \phi, \xi, \eta, g)$ shall be called quasi-conformally flat if the quasi-conformal curvature tensor $\widetilde{\mathrm{C}}=0$. It is known that the quasi-conformally flat manifold is either conformally flat if $a \neq 0$ or Einstein if $a=0$ and $b \neq 0[3]$. If the manifold $(M, \phi, \xi, \eta, g)$ satisfies $R(X, Y) . \widetilde{C}=0$, then the manifold is said to be quasi-conformally semisymmetric manifold. A manifold $(M, \phi, \xi, \eta, g)$ is said to be Ricci symmetric if R.S $=0$ holds on $M$ [7]. The class of Ricci semisymmetric manifolds includes the set of Ricci symmetric manifolds $(\nabla S=0)$ as a proper subset. Every semisymmetric manifold is Ricci symmetric. The converse is not true.

In the present paper quasi-conformally flat and quasi-conformally semisymmetric generalized Sasakian-space-forms are studied. The paper is organized as follows: Section 2 of this paper contains some preliminary results on generalized Sasakian-space-forms. In section 3, we study quasiconformally flat generalized Sasakian-space-forms and obtain necessary and sufficient conditions for a generlized Sasakian-space-form to be quasi-conformally flat. Also, we consider quasi-conformally Ricci tensor and quasi-conformally Ricci symmetric generalized Sasakian space-forms. In the next section, we deal with quasi-conformally semisymmetric generalized Sasakian-space-forms and it is proved that a generalized Sasakian-space-form is quasi-conformally semisymmetric if and only if the space-form is quasi-conformally flat and $f_{1}=f_{3}$.

\section{Preliminaries}

An odd-dimensional manifold $M^{2 n+1}$ is said to admit an almost contact structure $(\phi, \xi, \eta)$, if it carries a tensor field $\phi$ of type $(1,1)$, a vector field $\xi$ and a 1-form $\eta$ satisfying

$$
\phi^{2}=-\mathrm{I}+\eta \otimes \xi, \quad \eta(\xi)=1, \quad \phi \xi=0, \quad \eta \circ \phi=0 .
$$

If $\mathrm{g}$ is a compatible Riemannian metric with $(\phi, \xi, \eta)$ such that

$$
g(\phi X, \phi Y)=g(X, Y)-\eta(X) \eta(Y)
$$

or equivalently,

$$
g(X, \xi)=\eta(X), \quad g(X, \phi Y)=-g(\phi X, Y)
$$

for all vector fields $X, Y$ on $M^{2 n+1}$, then $M^{2 n+1}$ becomes an almost contact metric manifold with an almost contact metric structure $(\phi, \xi, \eta, g)$. An almost contact metric structure is called a contact metric structure if

$$
g(X, \phi Y)=\operatorname{d\eta }(X, Y)
$$


An almost contact metric manifold is Sasakian if and only if

$$
\left(\nabla_{X} \phi\right) Y=g(X, Y) \xi-\eta(Y) X
$$

for all vector fields $X, Y$ on $M^{2 n+1}$.

For a $(2 n+1)$-dimensional generalized Sasakian-space-form we have 1

$$
\begin{aligned}
R(X, Y) Z & =f_{1}\{g(Y, Z) X-g(X, Z) Y\} \\
& +f_{2}\{g(X, \phi Z) \phi Y-g(Y, \phi Z) \phi X+2 g(X, \phi Y) \phi Z\} \\
& +f_{3}\{\eta(X) \eta(Z) Y-\eta(Y) \eta(Z) X \\
& +g(X, Z) \eta(Y) \xi-g(Y, Z) \eta(X) \xi\}, \\
Q X & =\left(2 n f_{1}+3 f_{2}-f_{3}\right) X-\left(3 f_{2}+(2 n-1) f_{3}\right) \eta(X) \xi, \\
S(X, Y) & =\left(2 n f_{1}+3 f_{2}-f_{3}\right) g(X, Y)-\left(3 f_{2}+(2 n-1) f_{3}\right) \eta(X) \eta(Y), \\
r & =2 n(2 n+1) f_{1}+6 n f_{2}-4 n f_{3}
\end{aligned}
$$

for all vector fields X, Y, Z. By virtue of equations (7) and (9), we have

$$
\begin{aligned}
\eta(R(X, Y) Z) & =\left(f_{1}-f_{3}\right)\{g(Y, Z) \eta(X)-g(X, Z) \eta(Y)\} \\
R(X, Y) \xi & =\left(f_{1}-f_{3}\right)\{\eta(Y) X-\eta(X) Y\} \\
R(\xi, X) Y & =\left(f_{1}-f_{3}\right)\{g(X, Y) \xi-\eta(Y) X\} \\
S(X, \xi) & =2 n\left(f_{1}-f_{3}\right) \eta(X), \\
S(\xi, \xi) & =2 n\left(f_{1}-f_{3}\right) .
\end{aligned}
$$

The above results will be used in the next sections. Now we would like to recollect some of the examples of generalized Sasakian-space-forms.

Example 1: ( 11])A cosymplectic-space-form, i.e., a cosymplectic manifold with constant $\phi-$ sectional curvature $c$, is a generalized Sasakian-space-form with $f_{1}=f_{2}=f_{3}=c / 4$.

Example 2:( [9])A Kenmotsu-space-form, i.e., a Kenmotsu manifolds with constant $\phi$-sectional curvature $c$, is a generalized Sasakian-space-form with $f_{1}=(c-3) / 4$ and $f_{2}=f_{3}=(c+1) / 4$.

Example 3: (1])Let $N\left(F_{1}, F_{2}\right)$ be a generalized complex-space-form. Then, the warped product $M=\mathbb{R} \times_{f} N$, endowed with the almost contact metric structure $\left(\phi, \xi, \eta, g_{f}\right)$, is a generalized Sasakian-space-form $M\left(f_{1} ; f_{2} ; f_{3}\right)$ with functions:

$$
f_{1}=\frac{\left(F_{1} \circ \pi\right)-f^{\prime 2}}{f^{2}}, \quad f_{2}=\frac{F_{2} \circ \pi}{f^{2}}, \quad f_{3}=\frac{\left(F_{1} \circ \pi\right)-f^{\prime 2}}{f^{2}}+\frac{f^{\prime \prime}}{f} .
$$

In particular if $\mathrm{N}(\mathrm{c})$ is a complex-space-form, we obtain the generalized Sasakian-space-form

$$
M\left(\frac{c-4 f^{\prime 2}}{4 f^{2}}, \frac{c}{4 f^{2}}, \frac{c-4 f^{\prime 2}}{4 f^{2}}+\frac{f^{\prime \prime}}{f}\right) .
$$

Hence, the warped products $\mathbb{R} \times_{f} \mathbb{C}^{n}, \mathbb{R} \times_{f} \mathbb{C P}^{n}(4)$ and $\mathbb{R} \times_{f} \mathbb{C H}^{n}(-4)$ are generalized Sasakianspace-forms. 
Example 4: $\mathbb{R} \times_{f} \mathbb{C}^{m}$ is a generalized Sasakian-space-form with

$$
f_{1}=-\frac{\left(f^{\prime}\right)^{2}}{f^{2}}, \quad f_{2}=0, \quad f_{3}=-\frac{\left(f^{\prime} 1\right)^{2}}{f^{2}}+\frac{f^{\prime \prime}}{f},
$$

where $f=f(t)$.

\section{Quasi-conformally flat generalized Sasakian-space-forms}

If the generalized Sasakian-space-form $M\left(f_{1}, f_{2}, f_{3}\right)$ under consideration is quasi-conformally flat, then we have from (1)

$$
\begin{aligned}
R(X, Y, Z, W)= & \frac{b}{a}[S(X, Z) g(Y, W)-S(Y, Z) g(X, W) \\
& +S(Y, W) g(X, Z)-S(X, W) g(Y, Z)] \\
& +\frac{r}{(2 n+1) a}\left[\frac{a}{2 n}+2 b\right][g(Y, Z) g(X, W)-g(X, Z) g(Y, W)],
\end{aligned}
$$

where $a$ and $b$ are constants and $R(X, Y, Z, W)=g(R(X, Y) Z, W)$. Now putting $Z=\xi$ in (16) and using (4), (12) and (14) we get

$$
\begin{aligned}
& \left(f_{1}-f_{3}\right)[g(X, W) \eta(Y)-g(Y, W) \eta(X)] \\
= & 2 n\left(f_{1}-f_{3}\right) \frac{b}{a}[g(Y, W) \eta(X)-g(X, W) \eta(Y) \\
& +S(Y, W) \eta(X)-S(X, W) \eta(Y)] \\
& +\frac{r}{(2 n+1) a}\left[\frac{a}{2 n}+2 b\right][g(X, W) \eta(Y)-g(Y, W) \eta(X)] .
\end{aligned}
$$

Again putting $X=\xi$ in (17) and using (4) and (14) it follows that

$$
S(Y, W)=A g(Y, W)+B \eta(Y) \eta(W),
$$

where

$$
A=\left[-\frac{a}{b}\left(f_{1}-f_{3}\right)-2 n\left(f_{1}-f_{3}\right)+\frac{r}{(2 n+1) b}\left(\frac{a}{2 n}+2 b\right)\right]
$$

and

$$
B=\left[\frac{a}{b}\left(f_{1}-f_{3}\right)+4 n\left(f_{1}-f_{3}\right)-\frac{r}{(2 n+1) b}\left(\frac{a}{2 n}+2 b\right)\right] .
$$

Here $A+B=2 n\left(f_{1}-f_{3}\right)$. In the equation (18) putting $Y=W=\left\{e_{i}\right\}$, where $\left\{e_{i}\right\}$ is an orthonormal basis of the tangent space at each point of the manifold and taking summation over $i, 1 \leq i \leq 2 n+1$, we get

$$
r=(2 n+1) A+B .
$$

Now with the help of (19) and (20) the equation (21) gives

$$
[a+(2 n-1) b]\left[\frac{r}{2 n+1}-2 n\left(f_{1}-f_{3}\right)\right]=0 .
$$


If $a+(2 n-1) b=0$ and $a \neq 0 \neq b$. Then from (1) it follows that

$$
\widetilde{C}(X, Y) Z=a C(X, Y) Z \text {, }
$$

where $C(X, Y) Z$ denotes the Weyl conformal curvature tensor. But, under the consideration $\widetilde{C}=0$. So the quasi-conformal flatness and conformally flatness are equivalent. This implies that $\mathrm{C}=0$. If $a+(2 n-1) b \neq 0$ and $a \neq 0$. Then from (22) .

$$
r=2 n(2 n+1)\left(f_{1}-f_{3}\right) .
$$

So by comparing (10) and (24) we have

$$
3 f_{2}+(2 n-1) f_{3}=0
$$

By taking account of (25) in (9), we get

$$
S(X, Y)=2 n\left(f_{1}-f_{3}\right) g(X, Y) .
$$

This shows that, $M\left(f_{1}, f_{2}, f_{3}\right)$ is an Einstein. Thus we state the following:

Theorem 1. A quasi-conformally flat generalized Sasakian-space-from is either conformally flat or an Einstein manifold with scalar curvature $r=2 n(2 n+1)\left(f_{1}-f_{3}\right)$.

In the above theorem we have seen if $a+(2 n-1) b=0$ and $a \neq 0 \neq b$, then it follows that a quasi-conformally flat generalized Sasakian-space-form is conformally flat. But, it is known that [10] a $(2 n+1)$-dimensional $(n>1)$ generalized Sasakian-space-form $M\left(f_{1}, f_{2}, f_{3}\right)$ is conformally flat if and only if $f_{2}=0$. So in this case $M\left(f_{1}, f_{2}, f_{3}\right)$ is quasi-conformally flat if and only if $f_{2}=0$.

On the other hand, if $a+(2 n-1) b \neq 0$ and $a \neq 0$ then we have (24). By comparing the equations (10) and (24), one can get (25).

Conversely, suppose that (25) holds. Then in view of (7), (9) and (25), we can write the equation (1) as

$$
\begin{aligned}
& \widetilde{C}(X, Y, Z, W) \\
& =\frac{a}{1-2 n} f_{2}[g(Y, Z) g(X, W)-g(X, Z) g(Y, W)] \\
& +a f_{2}[g(X, \phi Z) g(\phi Y, W)-g(Y, \phi Z) g(\phi X, W)+2 g(X, \phi Y) g(\phi Z, W)] \\
& +\frac{3 a}{1-2 \eta} f_{2}[g(Y, W) \eta(X) \eta(Z)-g(X, W) \eta(Y) \eta(Z) \\
& +g(X, Z) \eta(Y) \eta(W)-g(Y, Z) \eta(X) \eta(W)]
\end{aligned}
$$

where $\widetilde{C}(X, Y, Z, W)=g(\widetilde{C}(X, Y) Z, W)$.

Replacing $X$ by $\phi X$ and $Y$ by $\phi Y$ in (27) we get

$$
\begin{aligned}
& \widetilde{C}(\phi X, \phi Y, Z, W) \\
& =\frac{a}{1-2 n} f_{2}[g(\phi Y, Z) g(\phi X, W)-g(\phi X, Z) g(\phi Y, W)] \\
& +\operatorname{af}_{2}\left[g(\phi X, \phi Z) g\left(\phi^{2} Y, W\right)-g(\phi Y, \phi Z) g\left(\phi^{2} X, W\right)+2 g\left(\phi X, \phi^{2} Y\right) g(\phi Z, W)\right] .
\end{aligned}
$$


Putting $Y=W=e_{i}$, where $\left\{e_{i}\right\}$ is an orthonormal basis of the tangent space at each point of the manifold, and taking summation over $i,(1 \leq i \leq 2 n+1)$, we get

$$
\begin{aligned}
& \sum_{i=1}^{2 n+1} \widetilde{C}\left(\phi X, \phi e_{i}, Z, e_{i}\right) \\
= & \frac{a}{2 n-1} f_{2} g(\phi X, \phi Z)+a f_{2}\left[-g(\phi X, \phi Z) g\left(\phi e_{i}, \phi e_{i}\right)+3 g\left(\phi^{2} X, \phi^{2} Z\right)\right] .
\end{aligned}
$$

Again putting $X=Z=e_{i}$, where $\left\{e_{i}\right\}$ is an orthonormal basis of the tangent space at each point of the manifold, and taking summation over $i,(1 \leq i \leq 2 n+1)$, we get after simplification $f_{2}=0$ with $a \neq 0$. Then in view of (25), we get $f_{3}=0$.

Therefore, we obtain from (7) that

$$
R(X, Y) Z=f_{1}\{g(Y, Z) X-g(X, Z) Y\}
$$

From (30) we have

$$
S(X, Y)=2 n f_{1} g(X, Y) \text { and } r=2 n(2 n+1) f_{1} .
$$

Hence in view of (1), we have $\widetilde{C}(X, Y) Z=0$. This leads to the following:

Theorem 2. Let $\mathrm{M}\left(\mathrm{f}_{1}, \mathrm{f}_{2}, \mathrm{f}_{3}\right)$ be a $(2 \mathrm{n}+1)$-dimensional $(\mathrm{n}>1)$ generalized Sasakian-space-form. Then $\mathrm{M}\left(\mathrm{f}_{1}, \mathrm{f}_{2}, \mathrm{f}_{3}\right)$ is quasi-conformally flat if and only if one of the following statements is true: (i) $\mathrm{a}+(2 \mathrm{n}-1) \mathrm{b}=0, \mathrm{a} \neq 0 \neq \mathrm{b}$ and $\mathrm{f}_{2}=0$.

(ii) $a+(2 n-1) b \neq 0, a \neq 0$ and $3 f_{2}+(2 n-1) f_{3}=0$.

In a $(2 n+1)$-dimensional $(n>1)$ manifold $(M, \phi, \xi, \eta, g)$, let $\left\{e_{i}\right\}, i=1,2, \ldots, 2 n+1$ be a local orthonormal basis. Then the quasi-conformal curvature tensor $\widetilde{C}(X, Y) Z$ defined as in (10), we can define a symmetric tensor of type $(0,2)$ called as quasi-conformal Ricci tensor and which is denoted by

$$
S_{\widetilde{C}}(X, Y)=\sum_{i=1}^{2 n+1} \widetilde{C}\left(e_{i}, X, Y, e_{i}\right),
$$

where $\sum_{i=1}^{2 n+1} \widetilde{C}\left(e_{i}, X, Y, e_{i}\right)=\sum_{i=1}^{2 n+1} g\left(\widetilde{C}\left(e_{i}, X\right) Y, e_{i}\right)$.

From (31) and (11), we have

$$
\left.S_{\widetilde{\mathrm{C}}}(X, Y)=\{a+(2 n-1) b\} S(X, Y)-\frac{r}{2 n+1} g(X, Y)\right\} .
$$

We first assume that a $(2 n+1)$-dimensional generalized Sasakian-space-form $M\left(f_{1}, f_{2}, f_{3}\right)$ is Ricci semisymmetric. That is,

$$
(R(X, Y) . S)(Z, W)=-S(R(X, Y) Z, W)-S(Z, R(X, Y) W)=0
$$

Now, since the curvature tensor $R$ of type $(0,4)$, defined by

$$
g(R(X, Y) Z, W)=R(X, Y, Z, W)
$$


is skew-symmetric where $R$ is the curvature tensor of type $(1,3)$, we get from (33) and (11) by taking account that $a+(2 n-1) b \neq 0$

$$
S_{\widetilde{C}}(R(X, Y) Z, W)+S_{\widetilde{C}}(Z, R(X, Y) W)=0
$$

which implies that

$$
\left(R(X, Y) . S_{\widetilde{C}}\right)(Z, W)=0 .
$$

So the space-form $M\left(f_{1}, f_{2}, f_{3}\right)$ is quasi-conformally Ricci semisymmetric.

Again, let us suppose that the space-form is quasi-conformally Ricci semisymmetric, that is, R.S $S_{\widetilde{\mathrm{C}}}=$ 0 holds in $M\left(f_{1}, f_{2}, f_{3}\right)$. Then (33) holds. Now using (33), and the skew-symmetric properties of $\mathrm{R}$ we get after simplification R.S $=0$, which implies that the space-form is Ricci semisymmetric. Hence the following theorem holds:

Theorem 3. $A(2 n+1)$-dimensional $(n>1)$ generalized Sasakian-space-form $M\left(f_{1}, f_{2}, f_{3}\right)$ is Ricci semisymmetric if and only if it is quasi-conformally Ricci semisymmetric provided that a + $(2 n-1) b \neq 0$.

\section{Quasi-conformally semisymmetric generalized Sasakian- space-forms}

In this section we consider a generalized Sasakian-space-form $M\left(f_{1}, f_{2}, f_{3}\right)$ satisfying the condition

$$
R(X, Y) \cdot \widetilde{C}=0 .
$$

Then we obtain from (11) by using (4), (12) and (14)

$$
\begin{aligned}
\eta(\widetilde{C}(X, Y) Z)= & \left\{(a+2 n b)\left(f_{1}-f_{3}\right)-\frac{r}{(2 n+1)}\left\{\frac{a}{2 n}+2 b\right\}\right\}[g(Y, Z) \eta(X) \\
& -g(X, Z) \eta(Y)]+b[S(Y, Z) \eta(X)-S(X, Z) \eta(Y)] .
\end{aligned}
$$

On taking $Z=\xi$ in the equation (35), we get

$$
\eta(\widetilde{C}(X, Y) \xi)=0 .
$$

Again putting $X=\xi$ in the equation (35), we have

$$
\begin{aligned}
\eta(\widetilde{C}(\xi, Y) Z)= & \left\{(a+2 n b)\left(f_{1}-f_{3}\right)-\frac{r}{(2 n+1)}\left\{\frac{a}{2 n}+2 b\right\}\right\}[g(Y, Z) \\
& -\eta(Y) \eta(Z)]+b\left[S(Y, Z)-2 n\left(f_{1}-f_{3}\right) \eta(Y) \eta(Z)\right] .
\end{aligned}
$$

In virtue of (34) we get

$$
\begin{aligned}
& R(X, Y) \widetilde{C}(U, V) W-\widetilde{C}(R(X, Y) U, V) W \\
& -\widetilde{C}(U, R(X, Y) V) W-\widetilde{C}(U, V) R(X, Y) W=0 .
\end{aligned}
$$


Which implies that

$$
\begin{aligned}
& \left(f_{1}-f_{3}\right)\{\widetilde{C}(U, V, W, Y)-\eta(Y) \eta(\widetilde{C}(U, V) W) \\
& +\eta(U) \eta(\widetilde{C}(Y, V) W)+\eta(V) \eta(\widetilde{C}(U, Y) W) \\
& +\eta(W) \eta(\widetilde{C}(U, V) Y)-g(Y, U) \eta(\widetilde{C}(\xi, V) W) \\
& -g(Y, V) \eta(\widetilde{C}(U, \xi) W)-g(Y, W) \eta(\widetilde{C}(U, V) \xi)\}=0 .
\end{aligned}
$$

Putting $\mathrm{U}=\mathrm{Y}$ in (39) and with the help of (35) and (36) we get either

$$
f_{1}=f_{3}
$$

or

$$
\begin{aligned}
& \{\widetilde{C}(Y, V, W, Y)+\eta(W) \eta(\widetilde{C}(Y, V) Y) \\
& -g(Y, Y) \eta(\widetilde{C}(\xi, V) W)-g(Y, V) \eta(\widetilde{C}(Y, \xi) W)\}=0 .
\end{aligned}
$$

Let $\left\{e_{1}, e_{2}, \ldots, e_{2 n+1}\right\}$ is an orthonormal basis of the tangent space at each point of the manifold. Putting $Y=e_{i}$ in (41) and taking summation over $i,(1 \leq i \leq 2 n+1)$, and using (35), (37) we get

$$
\left.S(V, W)=A^{\prime} g(V, W)+B^{\prime} \eta(V) \eta(W)\right\}
$$

where

$$
A^{\prime}=\frac{2 n(a+2 n b)\left(f_{1}-f_{3}\right)-r b}{a-b}
$$

and

$$
B^{\prime}=\frac{-2 n(2 n+1) b\left(f_{1}-f_{3}\right)+r b}{a-b} .
$$

Here $A^{\prime}+B^{\prime}=2 n\left(f_{1}-f_{3}\right)$. Now contracting (42) we get

$$
r=(2 n+1) A^{\prime}+B^{\prime} .
$$

By (43) and (44) the equation (45) gives

$$
(a+(2 n-1) b)\left(r-2 n(2 n+1)\left(f_{1}-f_{3}\right)\right)=0 .
$$

Therefore, either

$$
a+(2 n-1) b=0 \quad \text { or } \quad r=2 n(2 n+1)\left(f_{1}-f_{3}\right) .
$$

From (43) and (47) we obtain

$$
A^{\prime}=2 n\left(f_{1}-f_{3}\right) .
$$

By (44) and (47) we get

$$
\mathrm{B}^{\prime}=0
$$

So, from (42), (48) and (49) we have

$$
S(V, W)=2 n\left(f_{1}-f_{3}\right) g(V, W)
$$


Therefore, $M\left(f_{1}, f_{2}, f_{3}\right)$ is an Einstein manifold. Now with the help of (47) and (50) the equations (35) and (37) imply that

$$
\eta(\widetilde{C}(X, Y) Z)=0
$$

and

$$
\eta(\widetilde{C}(\xi, Y) Z)=0
$$

respectively. So using (36),(51) and (52) in (39) we get

$$
\widetilde{\mathrm{C}}(\mathrm{U}, \mathrm{V}, \mathrm{W}, \mathrm{Y})=0 .
$$

Therefore, by taking account of (40) and (53), we have either $f_{1}=f_{3}$ or $M\left(f_{1}, f_{2}, f_{3}\right)$ is quasiconformally flat.

Conversely, if $f_{1}=f_{3}$ then from (13) $R(\xi, U)=0$. Then obviously the condition $R(\xi, U) \cdot \widetilde{C}=$ 0 , that is, quasi-conformally semisymmetric condition is satisfied. Again if the space-form is quasi-conformally flat, then clearly it is quasi-conformally semisymmetric. Hence we conclude the following:

Theorem 4. $A(2 \mathrm{n}+1)$-dimensional $(\mathrm{n}>1)$ generalized Sasakian-space-form is quasi-conformally semisymmetric if and only if either the space-form is quasi-conformally flat or $\mathrm{f}_{1}=\mathrm{f}_{3}$.

By combining the Theorem 2 and Theorem 4, we can state the following corollary:

Theorem 5. Let $\mathrm{M}\left(\mathrm{f}_{1}, \mathrm{f}_{2}, \mathrm{f}_{3}\right)$ be a $(2 \mathrm{n}+1)$-dimensional $(\mathrm{n}>1)$ generalized Sasakian-space-form. Then $\mathrm{M}\left(\mathrm{f}_{1}, \mathrm{f}_{2}, \mathrm{f}_{3}\right)$ is quasi-conformally semisymmetric if and only if $\mathrm{f}_{1}=\mathrm{f}_{3}$ or one of the following statements is true:

(i) $\mathrm{a}+(2 \mathrm{n}-1) \mathrm{b}=0, \mathrm{a} \neq 0 \neq \mathrm{b}$ and $\mathrm{f}_{2}=0$.

(ii) $a+(2 n-1) b \neq 0, a \neq 0$ and $3 f_{2}+(2 n-1) f_{3}=0$.

It can be easily seen that $\nabla \mathrm{P}=0$ implies R.P $=0$. Hence by virtue of Theorem 4 we get

Corollary 4.1. A $(2 \mathrm{n}+1)$-dimensional $(\mathrm{n}>1)$ quasi-conformally symmetric generalized Sasakianspace-form is either quasi-conformally flat or $\mathrm{f}_{1}=\mathrm{f}_{3}$.

A Riemannian manifold is said to be quasi-conformally recurrent if $\nabla P=A \otimes P$, where $A$ is a non-zero 1-form. It can be easily shown that a quasi-conformally recurrent manifold satisfies $R \cdot P=0$. Hence we immediately get the following:

Corollary 4.2. A $(2 n+1)$-dimensional $(n>1)$ quasi-conformally recurrent generalized Sasakianspace-form is either quasi-conformally flat or $\mathrm{f}_{1}=\mathrm{f}_{3}$.

In particular, for Sasakian-space-form $f_{1}=\frac{c+3}{4}$ and $f_{3}=\frac{c-1}{4}$. So, $f_{1} \neq f_{3}$. Hence we can have the following corollary:

Corollary 4.3. $A(2 n+1)$-dimensional $(n>1)$ Sasakian-space-form is quasi-conformally semisymmetric if and only if it is quasi-conformally flat. 
Remark: If we take $f(t)=e^{t}$ in Example 4, we have $f_{1}=-1, f_{2}=0$ and $f_{3}=0$. Therefore, the condition $3 f_{2}+(2 n-1) f_{3}=0$ and $f_{2}=0$ holds. Hence from Theorem 2, generalized sasakianspace-form $\mathbb{R} \times_{f} \mathbb{C}^{m}$ with $f(t)=e^{t}$ is quasi-conformally flat.

Similarly from Theorem [5, generalized Sasakian-space-form $\mathbb{R} \times_{f} \mathbb{C}^{m}$ with $f(t)=e^{t}$ is quasiconformally semisymmetric.

Acknowledgement: The first author (DGP) is thankful to University Grants Commission, New Delhi, India for financial support in the form of Major Research Project F. No. 39-30/2010 (SR), dated: 23-12-2010.

\section{Received: May 2012. Accepted: September 2013.}

\section{References}

[1] P. Alegre, D. Blair and A. Carriazo, Generalized Sasakian-space-forms, Israel J. Math. 14 (2004), 157-183.

[2] P. Alegre and A. Carriazo, Structures on generalized Sasakian-space-form, Differential Geom. and its application 26 (2008), 656-666. doi. 10.1016/j difgeo.

[3] K. Amur and Y.B. Maralabhavi, On quasi-conformally flat spaces, Tensor (N.S.) 31 (2)(1977), 194-198. Math Soc., 45 (2)(2008), 313 - 319.

[4] D.E. Blair, Contact manifolds in Riemannian Geometry, Lecture Notes in Mathematics, Vol. 509, Springer-Verlag, Berlin, 1976.

[5] U.C. De and A. Sarkar, Some results on generalized Sasakian-space-forms, Thai J. Math. 8(1) (2010), 1-10.

[6] U.C. De and A. Sarkar, On the projective curvature tensor of generalized Sasakian-spaceforms, Quaestiones Mathematicae, 33(2)(2010), 245-252.

[7] R. Deszcz, On the equivalance of Ricci-semisymmetry and semisymmetry, Dept. Math. Agricultural Univ. Wroclaw, Preprint No.64, 1998.

[8] L.P. Eisenhart, Riemannian Geometry, Princeton University Press, princeton, N. J., 1949.

[9] K. Kenmotsu, A class of almost contact Riemannian manifolds, Tohoku Math.J., 24 (1972), 93-103.

[10] U.K. Kim, Conformally flat generalized Sasakian-space-forms and locally symmetric generalized Sasakian-space-forms, Note di matemetica 26 (2006), 55-67.

[11] L.D. Ludden, Submanifolds of cosymplectic manifolds, J.Diff Geom. 4 (1970), 237-244. 
[12] D.G. Prakasha, On generalized Sasakian-space-forms with Weyl-conformal curvature tensor, Lobacheviskii J. Math., 33 (3) (2012), 223-228.

[13] Z.I. Szabo, Structures theorems on Riemannian spaces satisfying $\mathrm{R}(\mathrm{X}, \mathrm{Y}) \cdot \mathrm{R}=0$, I. The local version J. Diff. Geom. 17 (1982), 531-582.

[14] K. Yano and S. Sawaki, Riemannian manifolds admitting a conformal transformation group, J. Diff Geom. 2 (1968), 161-184. 